\title{
Aprendizagem motora e síndrome de Down: efeitos da frequência relativa reduzida de conhecimento de resultados
}

\section{Motor learning and Down syndrome: effects of reduced relative frequency of knowledge of results}

\author{
Suzete Chiviacowsky ${ }^{1}$ \\ Camila Machado ${ }^{2}$ \\ Alexandre Carriconde Marques \\ José Francisco Gomes Schild ${ }^{1}$ \\ Ricardo Drews
}

Resumo - O objetivo do presente estudo foi comparar os efeitos de duas frequências (100 e 33\%) de conhecimento de resultados (CR) na aprendizagem de uma habilidade motora em indivíduos com síndrome de Down (SD). Vinte participantes com SD foram divididos, aleatoriamente, em dois grupos. O grupo 100\% recebeu CR após cada tentativa, enquanto o grupo $33 \%$ recebeu CR em um terço das tentativas, ou seja, uma tentativa com CR em cada bloco de três tentativas. A tarefa consistiu em arremessar um implemento em um alvo, com a mão dominante, sem visualizar o alvo. A fase de aquisição constou de 60 tentativas de prática, enquanto a fase de retenção, realizada 48 horas após, de 10 tentativas, sem CR. Os resultados indicaram a inexistência de diferença significativa entre os grupos. Conclui-se que frequências relativas reduzidas de CR podem ser tão efetivas quanto altas frequências para a aprendizagem de habilidades motoras em adultos com SD.

Palavras-chave: Arremesso; Comportamento motor; Deficiência intelectual; Feedback; Síndrome de down.

Abstract - The objective of the present study was to verify the effects of two frequencies (100 and 33\%) of knowledge of results (KR) on the learning of a motor skill, in individuals with Down syndrome (DS). Twenty participants with DS were randomly assigned into two groups. While the $100 \%$ group received KR after each trial, the 33\% group received KR in a third of the trials. The task consisted of throwing an implement on a target, with the dominant hand, while blindfolded. The acquisition phase consisted of 30 practice trials, while the retention phase, performed 48 hours later, consisted of 10 trials without KR. The results showed no differences between the groups. We concluded that reduced relative frequencies of $K R$ are as effective as high frequencies on the learning of simple motor tasks in adults with DS. Key words: Down syndrome; Feedback; Intellectual disability; Motor behavior; Throw.

1 Universidade Federal de Pelotas. Escola Superior de Educação Física. Pelotas, RS. Brasil

2 Universidade da Região da Campanha. Bagé, RS. Brasil

Recebido em 09/04/12 Revisado em 22/06/12 Aprovado em 27/07/12

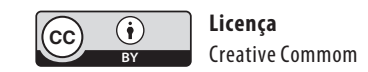




\section{INTRODUÇÃO}

Pessoas com síndrome de Down apresentam um grande espectro de diferenças em relação a pessoas sem síndrome de Down (SD). Causada pela trissomia do cromossoma $21^{1}$, a SD está associada a mudanças na anatomia corporal e no desenvolvimento cognitivo, resultando em padrões de movimentos atípicos ${ }^{2,3}$. Ainda, o controle postural e a competência perceptivo-motora em crianças com SD é normalmente atrasada quando comparada a crianças e adolescentes sem a síndrome ${ }^{4,5}$.

Programas de atividade física aparentam possuir o potencial para afetar positivamente a saúde geral de adultos com a SD, aumentando a sua qualidade de vida ${ }^{6}$. De fato, a expectativa de vida das pessoas com SD tem aumentado significativamente, em decorrência de avanços recentes em tratamento médico e de inclusão social ${ }^{7}$, com estudos indicando uma expectativa de vida de mais de 50 anos de idade ${ }^{8}$.

Considerando que movimentos habilidosos são fundamentais à experiência humana, a análise de como a aprendizagem motora pode ser otimizada em pessoas com SD não possui apenas interesse teórico, mas também implicações práticas importantes para a vida destas pessoas. Um fator que consistentemente tem demonstrado beneficiar a aprendizagem de habilidades motoras em sujeitos típicos é a frequência reduzida de conhecimento de resultados (CR).

Estudos com participantes típicos ${ }^{9-13}$, ou que apresentam algum grau de deficiência motora como, por exemplo, a doença de Parkinson ${ }^{14}$, têm demonstrado que frequências reduzidas de CR podem beneficiar a aprendizagem de habilidades motoras.

Tais resultados indicam que certa quantidade de informação em forma de CR é necessária para que a melhora da performance ocorra, mas se a quantidade for excessiva, os participantes podem apresentar a tendência de depender muito dele e negligenciar a aprendizagem de outros aspectos relevantes à tarefa através da utilização das informações fornecidas pelo feedback intrínseco, importantes para o desenvolvimento da capacidade de detecção e correção de erros ${ }^{15}$.

Parece, assim, que a frequência reduzida de CR ébenéfica à aprendizagem de habilidades motoras em adultos típicos. Entretanto, pouco se sabe sobre os possíveis efeitos desta variável na aprendizagem de habilidades motoras em populações com necessidades especiais, mais especificamente em adultos com SD. Um fator que possui o potencial de limitar a generalização destes efeitos é a deficiência intelectual e motora desta população. Alguns estudos têm procurado examinar as diferenças no controle e na coordenação motora entre participantes típicos e participantes com SD. Por exemplo, Robertson et al. ${ }^{16}$ demonstraram diferenças na coordenação de um padrão de movimento específico entre estes dois grupos, com participantes com SD, utilizando menos tempo no modo de coordenação "in-phase" e mais tempo nos modos "anti-phase" e "intermediate-phase", sendo estes últimos considerados como modos menos estáveis de coordenação, do que os participantes típicos. 
Também Latash et al. ${ }^{17}$ encontraram diferenças entre participantes típicos e com SD, como picos de força reduzidos e produção involuntária de força por musculatura que não deveria estar envolvida em certos movimentos. Tais resultados podem indicar uma maior dificuldade de processamento de feedback intrínseco relacionado à tarefa, em pessoas com SD, com consequente maior necessidade de feedback extrínseco, a fim de obter melhores resultados durante o processo de aprendizagem de uma habilidade motora.

De fato, de acordo com Bogaertz e Swinnen ${ }^{18}$, pessoas com SD necessitam de uma carga maior de atenção a fim de monitorar aspectos básicos da performance motora, devido à menor capacidade de controlar movimentos, aparentando, assim, possuir recursos limitados de atenção para processar informações adicionais. Em um estudo que verificou o processamento de feedback visual em movimentos direcionados a um alvo em pessoas com $\mathrm{SD}$, Hodges et al. ${ }^{19}$ demonstraram que os participantes com SD exibiram tempos de movimento mais longos que os participantes típicos, sugerindo serem aqueles mais dependentes do feedback produzido pela resposta ou requererem mais tempo para processar o feedback. Tais resultados sugerem, de forma mais específica, que diferente de sujeitos típicos, as pessoas com SD podem necessitar de mais informação extrínseca durante a aprendizagem de uma determinada tarefa, com frequências reduzidas de CR não sendo tão benéficas à aprendizagem motora desta população.

Até o presente momento, poucos estudos tiveram a preocupação de compreender os mecanismos e os fatores que afetam a aprendizagem de habilidades motoras em pessoas com SD. Os efeitos positivos do feedback visual e auditivo aumentado têm sido demonstrados no contexto do tratamento das desordens motoras da fala. Por exemplo, crianças e adolescentes com SD demonstraram melhora na precisão da produção da fala quando lhes foi fornecido feedback visual, baseado em equipamentos de eletropalatografia ${ }^{20,21}$. Os resultados demonstram a importância desta variável para a aprendizagem de habilidades motoras em sujeitos com a síndrome e tornam clara a necessidade de estudos que procurem examinar os efeitos de diferentes variações de fornecimento do feedback extrínseco sobre a aprendizagem.

Tendo em vista os efeitos positivos da frequência reduzida de CR para a aprendizagem de habilidades motoras ${ }^{10,11,14}$, o objetivo deste estudo é examinar se os benefícios desta variável podem ser generalizados a sujeitos com SD. Espera-se que a frequência reduzida de CR beneficie a aprendizagem de habilidades motoras nesta população.

\section{PROCEDIMENTOS METODOLÓGICOS}

\section{Amostra}

Participaram do presente estudo vinte adultos (8 homens e 12 mulheres) com SD, entre 19 e 27 anos de idade (média de idade 21.5; DP =2.25), participantes de um projeto de extensão da universidade. Todos os participantes foram diagnosticados com SD e deficiência intelectual média (faixa de QI: 50 - 70), através da escala de Stanford-Binet. Como critérios de exclusão fo- 
ram considerados: um nível diferente de deficiência intelectual, diagnóstico de autismo ou outra desordem neurológica. Todos os sujeitos participaram como voluntários e sua participação foi decidida após assinatura do termo de consentimento por parte dos pais ou responsáveis, tendo o estudo sido aprovado pelo comitê de ética da Universidade Federal de Pelotas, com protocolo número 088/2009. Os participantes não possuíam conhecimento sobre o objetivo do experimento, nem experiência anterior com a tarefa.

\section{Tarefa}

Foi utilizado um alvo circular impresso em pano e afixado no solo, com $2 \mathrm{~m}$ de diâmetro, com o seu centro à uma distância de $4 \mathrm{~m}$ da área de arremesso. A tarefa consistiu em arremessar, com a mão dominante e sem auxílio da visão, saquinhos de feijão (pesando 100 gramas) em um alvo. O centro do alvo mediu $20 \mathrm{~cm}$ e teve o valor 100; os outros espaços tiveram respectivamente $10 \mathrm{~cm}$ a mais de raio, com valores $90,80,70,60,50,40,30,20,10 \mathrm{e}$ zero. Ainda, o alvo foi dividido em duas partes (antes ou depois), onde foi possível obter a direção do erro, sendo o CR também fornecido em termos de distância do centro do alvo, ou seja, o experimentador também indicava se o implemento havia pousado "um pouco" (círculos de 60 a 100) ou "muito" (de 0 ao círculo 50) longe do alvo. Um óculos de natação adaptado, ou seja, com as lentes cobertas por material opaco, foi utilizado a fim de impedir a visualização dos resultados por parte dos sujeitos durante as tentativas.

\section{Procedimentos}

A amostra foi distribuída em dois grupos: 10 sujeitos para o grupo que recebeu $100 \%$ de frequência de CR (grupo 100\% CR) e 10 sujeitos para o grupo que recebeu 33\% de frequência de CR (grupo 33\% CR). Os participantes foram distribuídos entre os grupos com base na ordem cronológica de participação, com o cuidado de igualar o número de homens e mulheres em cada grupo. O grupo 100\% CR recebeu CR após cada tentativa, enquanto o grupo $33 \%$ CR recebeu CR em um terço das tentativas, ou seja, uma com CR, duas sem CR e assim sucessivamente. O número de tentativas para a fase de aquisição foi determinado após a realização de um estudo piloto, a fim de verificar o número de tentativas necessárias para alcançar a estabilização, ou seja, o número de tentativas em que o desempenho dos participantes praticamente estabiliza o seu processo de diminuição de erro; neste caso, 60. A fase de retenção foi realizada 48 horas após a fase de aquisição, composta por 10 tentativas, sem CR.

Os participantes foram informados sobre o objetivo da tarefa. Foi solicitado que se posicionassem confortavelmente no local designado para os arremessos, em frente ao alvo. O experimentador demonstrou a tarefa uma vez durante a instrução, para que se familiarizassem com a forma correta de realizar os arremessos e assegurou-se da correta compreensão, por parte dos participantes, tanto das instruções quanto do feedback a ser fornecido, solicitando aos mesmos que verbalmente repetissem o que seria feito. Após as instruções, os participantes colocaram os óculos de natação adaptado, a fim 
de impedir o feedback visual. Enquanto o grupo $100 \%$ CR foi informado de que feedback após cada tentativa, os participantes do grupo 33\% CR foram informados de que receberiam feedback após algumas tentativas apenas.

\section{Análise dos dados}

A variável dependente foi o Erro Absoluto (em $\mathrm{cm}$ ). Os dados da fase de aquisição foram analisados através da análise de variância (ANOVA) two-way: 2 (grupo: 100\% CR versus 33\% CR) x 6 (blocos de 10 tentativas), com medidas repetidas no último fator, após o teste de Shapiro-Wilk demonstrar a normalidade dos dados. Já os dados da fase de retenção foram analisados através da ANOVA one-way 2 (grupo: 100\% CR versus 33\% CR).

\section{RESULTADOS}

\section{Fase de Aquisição}

Pode ser observado (Figura 1, blocos A1 a A6) que ambos os grupos melhoraram o seu desempenho, de forma constante e similar, durante os três primeiros blocos da fase de aquisição, mantendo-se estáveis do terceiro para o quarto e quinto blocos, piorando no último bloco de tentativas. Observa-se também uma tendência de menor valor de erro do grupo 100\% CR no bloco A1 em relação ao grupo 33\%. Através da ANOVA two-way, foram encontradas diferenças significativas entre os blocos, $F(5 ; 90)=4,20, p=$ $0,002, \eta^{2}=.19$, mas não entre os grupos $F(1 ; 18)=0,04, p=0,83$, ou na interação entre blocos e grupos $F(5 ; 90)=0,27, p=0,92$.

Fase de Retenção. Para a fase de retenção (Figura 1, bloco R), pode-se constatar a inexistência de diferença no desempenho dos grupos, confirmada pela ANOVA One-Way $F(1,19)=0,10, p=0,75$.

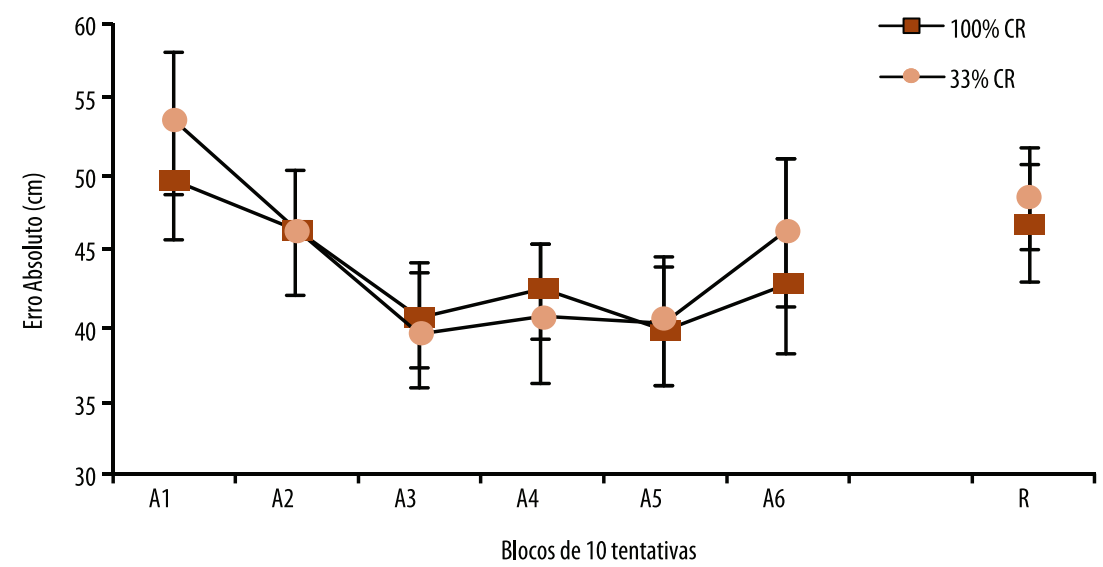

Figura1. Médias dos grupos, em erro absoluto, nas fases de aquisição e retenção. As barras de erro representam a variabilidade dos grupos em forma de erro padrão.

\section{DISCUSSÃO}

Estudos que procurem verificar os efeitos dos fatores que afetam a aprendizagem motora na área da reabilitação e da atividade motora adaptada são 
ainda escassos ${ }^{22-24}$. O objetivo do presente estudo foi verificar os efeitosde duas frequências de $\mathrm{CR}$, controladas pelo experimentador, em sujeitos adultos com SD. Adultos com SD diferem de adultos típicos tanto em relação a aspectos $\operatorname{cognitivos}^{3}$ quanto de performance motora ${ }^{4,5,25}$. Assim, ainda não era claro se os benefícios de aprendizagem demonstrados em sujeitos típicos ${ }^{9,11-13}$ ou com a doença de Parkinson ${ }^{14}$, que recebem frequências reduzidas de $\mathrm{CR}$, serão similares nesta população, ou se a frequência reduzida de CR poderá prejudicar a aprendizagem dos mesmos quando comparada a uma frequência de $100 \%$ de CR.

Os resultados do presente estudo mostraram que os participantes com SD obtiveram aprendizagem similar tanto em um arranjo de prática com frequência reduzida a $33 \%$ de CR quanto com um arranjo com frequência de $100 \%$ de CR. Tais resultados confirmam os estudos prévios sobre o tema, ao demonstrarem que frequências reduzidas podem ser tão benéficas ou superiores à aprendizagem de habilidades motoras, quanto frequências aumentadas ${ }^{09-14}$.

A hipótese de orientação ou "guidance hypothesis" colocada por Salmoni et al. ${ }^{15}$, procura explicar os efeitos positivos da frequência reduzida de CR, sendo uma das mais aceitas na área da aprendizagem motora. Esta hipótese faz referência à forte função informacional do CR, cujas propriedades são capazes de guiar ou de orientar os aprendizes em direção ao objetivo da tarefa. A informação de CR pode ser considerada como um auxiliar externo para a redução do erro, principalmente, no início da aprendizagem. Nesta fase, os aprendizes apresentam mais dificuldade em obter sucesso durante as tentativas de prática, em perceber ou detectar as características do erro cometido e mesmo em realizar processos de resgate e elaboração do plano de ação, necessários para o desempenho da próxima tentativa. Desta forma, torna-se muito mais fácil ao sujeito, nesta fase de aprendizagem, utilizar as informações extrínsecas, prontamente disponíveis e sempre corretas relacionadas ao erro executado, do que, esforçadamente, ter que processar informações por conta própria. No entanto, como resultado, pode ocorrer um processamento insuficiente de informações intrínsecas por parte do aprendiz. De acordo com Salmoni et al. ${ }^{15}$ tal processamento é considerado importante para o desenvolvimento de capacidades de detecção e correção de erros, fundamentais para a aprendizagem da tarefa e consequente manutenção do desempenho em situações nas quais o feedback extrínseco não se encontra mais a disposição, como normalmente acontece nas fases de retenção e/ou transferência. Outra explanação relacionada aos efeitos da frequência de CRé a hipótese da instabilidade ou "maladaptive short-term corrections", colocada por Schmidt ${ }^{26}$. Uma excessiva instabilidade ou falta de consistência durante a prática pode ocorrer quando informações de CR são fornecidas de forma muito frequente, pois provoca correções sucessivas do desempenho, mesmo em erros muito pequenos. De acordo com o autor, tais correções sucessivas causadas pelo feedback frequente podem dificultar o desenvolvimento da capacidade de manifestar estabilidade na retenção e na transferência.

Entretanto, tais hipóteses não são capazes de explicar as similaridades de comportamento encontradas nas diferentes fases do presente experi- 
mento, ou seja, o grupo que recebeu $100 \%$ de CR não demonstrou ter ficado dependente da informação extrínseca na fase de retenção e não teve sua aprendizagem prejudicada por possíveis instabilidades provocadas pela frequência aumentada, em relação ao grupo que recebeu frequência reduzida.

Por outro lado, pode-se questionar a eficiência da frequência reduzida utilizada no presente estudo (33\% de CR) para aprendizes que apresentam deficiência cognitiva e perceptivo-motora. Estudos realizados com sujeitos com a doença de Parkinson têm demonstrado resultados conflitantes em relação às diferentes frequências utilizadas. Por exemplo, o estudo de Guadagnoli et al. ${ }^{27}$ comparou frequências de CR de $20 \%$ e $100 \%$ na aprendizagem de uma tarefa motora de posicionamento, com os resultados demonstrando uma interação significativa entre sujeitos típicos e com a doença de Parkinson, com maior aprendizagem para o grupo que recebeu $20 \%$ de CR em sujeitos típicos e, ao contrário, maior aprendizagem para os sujeitos com a doença de Parkinson que receberam frequência de $100 \%$ de CR. Já o estudo de Chiviacowsky et al. ${ }^{14}$, comparando frequências de $66 \%$ e $100 \%$ de CR, encontrou resultados superiores de aprendizagem para uma frequência não tão reduzida de $\mathrm{CR}$ em comparação com o feedback fornecido após todas as tentativas.

Este estudo parece ser o primeiro a demonstrar os efeitos de diferentes frequências de CR na aprendizagem motora de sujeitos com SD. Tais resultados são importantes, pois são capazes de fornecer subsídios para a atuação de profissionais da fisioterapia ou da educação física onde, em sessões de aprendizagem motora, geralmente tendem a fornecer feedback extrínseco de forma muito frequente. O CR frequente, como os resultados do presente estudo demonstram, não é necessário.

\section{CONCLUSÃO}

Conclui-se que a frequência reduzida de 33\% de CR é tão benéfica à aprendizagem de uma habilidade motora simples com demanda espacial, em sujeitos com a SD quanto a frequência de $100 \%$. A realização de estudos com diferentes tarefas e frequências de CR (uma limitação do presente estudo) poderia auxiliar na maior compreensão dos efeitos desta importante variável de aprendizagem motora nesta população.

\section{REFERÊNCIAS BIBLIOGRÁFICAS}

1. Hassold T, Abruzzo M, Adkins K, Griffin D, Merrill M, Millie E, et al. Human aneuploidy: incidence, origin, and etiology. Environ Mol Mutagen 1996;28:167-75.

2. Latash ML. Learning motor synergies by persons with Down syndrome. J Intellect Disabil Res 2007;51:962-71.

3. Silverman W. Down syndrome: cognitive phenotype. Ment Retard Dev Disabil Res Rev 2007;13:228-36.

4. Meneghetti CHZ, Blascovi-Assis SM, Deloroso FT, Rodrigues GM. Static balance assessment among children and adolescents with Down syndrome. Rev Bras Fisioter 2009;13:230-5.

5. Spanò M, Mercuri E, Randò T, Pantò T, Gagliano A, Henderson S, et al. Motor and perceptual-motor competence in children with Down syndrome: variation in performance with age. Eur J Paediatr Neurol 1999;3:7-13. 
6. Barnhart RC, Connolly B. Aging and Down syndrome: implications for physical therapy. Phys Ther 2007;87:1399-406.

7. Wiseman FK, Alford KA, Tybulewicz VL, Fisher EM. Down syndrome - recent progress and future prospects. Hum Mol Genet 2009;15:75-83.

8. Glasson EJ, Sullivan SG, Hussain R, Petterson BA, Montgomery PD, Bittles AH. The changing survival profile of people with Down's syndrome: implications for genetic counselling. Clin Genet 2002;62:390-3.

9. Chiviacowsky S, Tani G. Efeitos da frequência do conhecimento de resultados na aprendizagem de uma habilidade motora em crianças. Rev Paul Educ Fís 1993;7:45-57.

10. Oliveira DL, Gimenez R, Correa UC, Basso L, Tani G. Relative frequency of knowledge of results and task complexity in the motor skill acquisition. Percept Mot Skills 2009;109:831-40.

11. Winstein CJ, Schmidt RA. Reduced frequency of knowledge of results enhances motor skill learning. J Exp Psychol: Learn Mem Cogn 1990;16:677-91.

12. Wulf G, Schmidt RA. The learning of generalized motor programs: Reducing the relative frequency of knowledge of results enhances memory. J Exp Psychol Learn Mem Cogn. 1989;15:748-57.

13. Wulf G, Schmidt RA, Deubel H. Reduced feedback frequency enhances generalized motor programs learning but not parameterization learning. J Exp Psychol Learn Mem Cogn. 1993;19:1134-50.

14. Chiviacowsky S, Campos T, Domingues MR. Reduced frequency of knowledge of results enhances learning in persons with Parkinson's disease. Front Psychol 2010;1:226. doi: 10.3389/fpsyg.2010.00226

15. Salmoni A, Schmidt RA, Walter CB. Knowledge of results and motor learning: A review and critical reappraisal. Psychol Bull 1994;95:355-86.

16. Robertson SD, Van Gemmert AW, Maraj BK. Auditory information is beneficial for adults with Down syndrome in a continuous bimanual task. Acta Psychol (Amst) 2002;110:213-29.

17. Latash ML, Kang N, Patterson D. Finger coordination in persons with Down syndrome: atypical patterns of coordination and the effects of practice. Exp Brain Res 2002;146:345-55.

18. Bogaerts H, Swinnen SP. Spatial interactions during bimanual coordination patterns: the effect of directional compatibility. Motor Control 2001;5:183-99.

19. Hodges NJ, Cunningham SJ, Lyons J, Kerr TL, Elliott D. Visual feedback processing and goal-directed movement in adults with Down Syndrome. Adapt Phys Activ Q 1995;12:176-86.

20. Cleland J, Timmins C, Wood SE, Hardcastle WJ, Wishart JG. Electropalatographic therapy for children and young people with Down's syndrome. Clin Linguist Phon 2009;23:926-39.

21. Wood S, Wishart J, Hardcastle W, Cleland J, Timmins C. The use of electropalatography (EPG) in the assessment and treatment of motor speech disorders in children with Down's syndrome: evidence from two case studies. Dev Neurorehabil 2009;12:66-75.

22. Vliet, PM, Wulf G. Extrinsic feedback for motor learning after stroke: What is the evidence? Disabil Rehabil 2006;28:831-40.

23. Wulf G. Self-controlled practice enhances motor learning: Implications for physiotherapy. Physiother 2007;93:96-101.

24. Wulf G, Shea CH, Lewthwaite R. Motor skill learning and performance: A review of influential factors. Med Edu 2010;44:75-84.

25. Gomes MM, Barela JA. Postural control in down syndrome: the use of somatosensory and visual information to attenuate body sway. Motor Control 2007;11:224-34.

26. Schmidt RA. Frequent augmented feedback can degrade learning: Evidence and interpretations. In Requin, J, Stelmach GE, editor. Tutorials in motor neuroscience Dordrecht, The Netherlands: Kluwer Academic Publishers; 1995. p. 59-75.

27. Guadagnoli MA, Leis B, Van Gemmert AW, Stelmach GE. The relationship between knowledge of results and motor learning in Parkinsonian patients. Parkinsonism Relat Disord 2002;9:89-95.

\section{Endereço para correspondência}

Suzete Chiviacowsky Escola Superior de Educação Física Universidade Federal de Pelotas Rua Luís de Camões, 625 CEP 96055-630 - Pelotas, RS - BRASIL E-mail: schivi@terra.com.br 